\title{
o $3 x y$ \\ Impact of COVID-19 on materials science research innovation and related pandemic response
}

\author{
Crystal Chu, Salmaan Baxamusa, and Claire Witherel, Guest Editors*
}

\begin{abstract}
The scope of impact that the coronavirus SARS-CoV-2 has had and continues to have on life, society, and the world as we know it will be debated for years to come. One thing is for certain, scientists, engineers, clinicians, and researchers around the globe rallied to heed the call for innovation, particularly in the field of materials science. In this special issue of MRS Bulletin, we feature six articles, two of which showcase primary consumable materials research and development, along with four review articles highlighting materials innovation over the last 18 months in diagnostics, prevention, and treatment of SARS-CoV-2 infection.
\end{abstract}

\section{Introduction}

Since the World Health Organization declared a Public Health Emergency of International Concern in January 2020 and subsequently a pandemic in March 2020, daily life the world over has been reorganized around efforts to battle COVID-19, the disease caused by the coronavirus SARS-CoV-2. In this issue of MRS Bulletin, we highlight the research response of the materials science community in response to the COVID-19 pandemic.

It is difficult to put into perspective the scale of this historic event when the event itself is ongoing. Reciting up-tothe-minute case counts and death tolls is de rigueur in popular, journalistic, and scientific communication. We will limit the reporting of such numbers here, but we will note that the global death toll is likely to be at least $0.05 \%$ of the world population. ${ }^{1}$

The global reach of the COVID-19 pandemic has thrust rapid scientific development-from bench to market-onto the critical path for limiting casualties and for resuming the normal functions of daily life the world over. From the early days of the pandemic, it became clear that the response from the research community would play an important role in slowing transmission, detecting, and treating COVID-19. Scientists, doctors, and engineers of all disciplines revectored their research and applied their expertise to addressing critical knowledge gaps and developed COVID-19-relevant technologies; materials science has been no exception. This special issue covers the role of materials-focused innovation in combatting the COVID-19 pandemic within three major categories, including consumables, diagnostics, to treatments and prevention strategies first in consumables. Novel primary research from Tooker et al. using $3 \mathrm{D}$ printing to create nasopharyngeal swabs and Wang et al. generating new filter media used in filter facepiece respirators (i.e., N95, KN95, etc.) are featured in this special issue. ${ }^{2,3}$ Additional reviews include extensive overviews in the area of materials for diagnostics research, including serological assays by Sadtler et al. and use of electrochemical biosensors by Furst et al. that were investigated as a result of the COVID-19 pandemic. ${ }^{4,5}$ Finally, Wayne et al. provide a robust historical context of materials science innovation in this pandemic, currently used therapies in the clinic, and an introduction to vaccine development, while Fenton et al. provide a thorough overview of the long-term research regarding lipid nanoparticles that has led to the novel vaccine technologies used by Moderna and Pfizer-BioNTech that have been deployed in millions of patients worldwide (Figure 1) ${ }^{6,7}$ These topical areas cover the breadth of technological maturity, from early research that lays important groundwork for future pandemic preparedness to process

Crystal Chu, Department of Chemistry, Lehigh University, USA; ckc221@lehigh.edu

Salmaan Baxamusa, Lawrence Livermore National Laboratory, USA; baxamusa1@|Inl.gov

Claire Witherel, Department of Bioengineering, University of Pennsylvania, USA; witherel@seas.upenn.edu

${ }^{*}$ Corresponding author

doi:10.1557/s43577-021-00186-1 


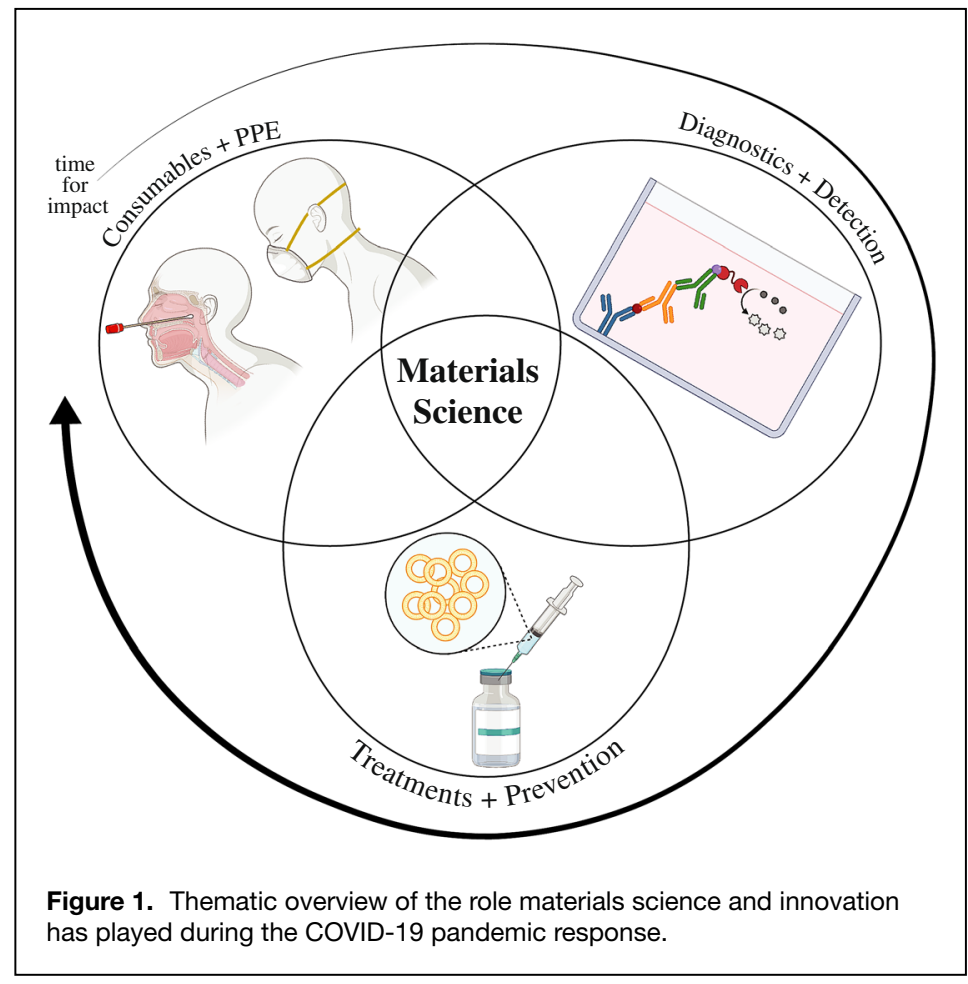

development for consumer goods and medical devices/therapeutics that are now on the market.

\section{Consumables materials development}

The COVID-19 pandemic created a surge in the demand for medical consumables far beyond the typical steady-state. It quickly became apparent that consumables would rapidly experience shortages unless improvements in manufacturing capacity could be immediately implemented. In addition to increases in manufacturing throughput - no small feat of its own-materials innovation was needed to strengthen supply

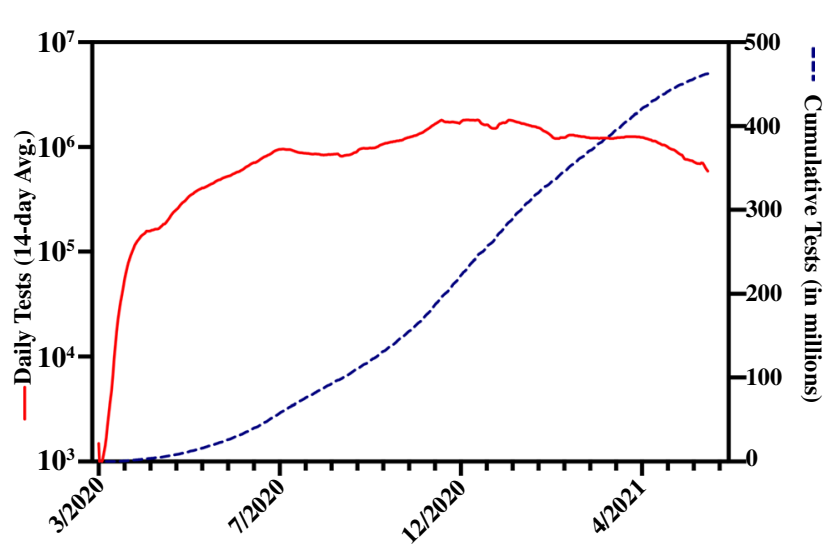

Figure 2. Fourteen-day average of daily tests (left axis) and cumulative tests (right axis) from March 1, 2020 through June 2021. chains by decentralizing manufacturing, repurposing existing tools, and developing and qualifying new materials.

Two consumables that experienced unprecedented increases in demand are nasopharyngeal (NP) swabs used for COVID-19 diagnostic testing and filter-based personal protective equipment (PPE) needed by health care professionals and, increasingly, desired by the general public. In this issue, research in NP swabs (Tooker et al.) and filter media (Wang et al.) are surveyed as materials science contributions to fighting the pandemic.

Early in the pandemic, health authorities emphasized the importance of widespread testing to identify and isolate COVID-19 positive patients as a means of slowing the spread of the virus. ${ }^{8}$ Figure 2 shows the number of COVID-19 tests performed in the United States over the course of the pandemic. ${ }^{9}$ In March 2020, when the first stayat-home guidelines were announced in the United States, the number of daily tests increased from 1000 to 100,000 . By July 2020, more than 1 million tests were being conducted in the United States daily, and as of June 2021, more than 450 million tests have been administered.

The gold-standard test for COVID-19 is reverse transcription polymerase chain reaction (RT-PCR) based detection of viral RNA collected via NP swabs. ${ }^{10}$ The NP swabs collect secretions from the back of the nose and throat on a flocked plastic tip, typically nylon. The massive increase in diagnostic demand strained all aspects of the testing chain. Shortages of NP swabs were identified as an early challenge to increasing testing capacity, particularly with disruption in traditional supply chains; three-dimensional (3D) printing was identified as a method both to reduce the development cycle time for new swab designs, and to decentralize production based on the wide availability of consumer and commercial 3D printers. In this issue, Tooker et al. describes the development of bench-top mechanical tests to evaluate swab functionality, safety, and comfort - particularly for novel 3D-printed design, which has subsequently been used by at least one manufacturer to improve NP swab design.

Another consumable that experienced a surge in demand as a result of the COVID-19 pandemic was PPE for health care professionals. Because the SARS-CoV-2 virus is primarily transmitted through respiratory droplets and aerosols, ${ }^{11}$ one of the most urgent early (and consistent) needs in medical settings was filter facepiece respirators (FFRs) capable of capturing airborne viral particles. FFRs contain a filter media, typically melt-blown polypropylene electrostatically charged by corona discharge, contained in a housing that snugly fits the wearer so that all aerosols must pass through the filter media. Such FFRs are commonly known as N95s, after the United States National Institute of Occupational 
Safety and Health (NIOSH) standard of filtering 95\% of $0.3-$ $\mu \mathrm{m}$ aerosolized particles. ${ }^{12}$ The N95 designation is comparable to other international designations such as KN95 (China) and FFP2 (European Union). ${ }^{13}$

N95 FFRs for medical use are normally meant to be disposable after a single use, especially when used in aerosolgenerating medical procedures such as intubation. However, shortages of N95 FFRs led to crisis conservation methods, and reuse of FFRs became common practice. In the spring of 2020, several studies reported methods to decontaminate or reduce the bioburden of used FFR filter media without degrading the overall form and fit of the filter housing itself. ${ }^{14-16}$ Vapor hydrogen peroxide and heat/humidity based methods, among others, were granted emergency use authorization by the United States Food and Drug Administration (FDA) in order to facilitate reuse of N95 FFRs, ${ }^{17}$ and large scale trials of N95 FFR reuse were reported in India. ${ }^{18}$ In April of 2021, in response to a stabilizing supply chain, the FDA recommended that United States health care facilities transition away from conservation strategies such as decontamination and reuse of disposable N95 FFRs. ${ }^{19}$

The development of inherently antiviral filter media or ceramic filters, both of which may lead to PPE that may be reused many times, is another long term goal of filter media materials research. In this issue, Wang et al. review the performance of common filtration fabrics, the development of decontamination protocols for N95 FFRs, and the longer-term outlook for a novel filter media and manufacturing methods. Such advances are expected to contribute to global preparedness for continuing to combat this and future pandemics. For instance, the work of Paranthaman, Theodore, and colleagues (described in the contribution by Wang et al.) was implemented by DemeTech Corporation of Florida (USA) to manufacture NIOSH-approved face coverings and FFRs that are now available to consumers.

Both the contributions on NP swab development (Tooker et al.) and filter media research (Wang et al.) have their origins in the National Virtual Biotechnology Laboratory (NVBL), a consortium of United States Department of Energy laboratories formed in March 2020. ${ }^{20}$ The NVBL is one of the many formal and informal organizations formed to coordinate a scientific response between governments, academia, health care facilities, and industry. Atypically for the MRS Bulletin, these two contributions contain a significant amount of previously unpublished research. The guest editors, with the support of the editorial board, have elected to include theses contributions in order to highlight materials research quickly initiated and brought into practice to respond to critical societal needs during the pandemic.

\section{Role of materials in diagnostics and detection}

The development of assays to effectively diagnose SARSCoV-2 infections has been a major effort in materials research in response to the COVID-19 pandemic. Prior to the authorization and administration of vaccines, social distancing and quarantining guidelines paired with diagnostic testing comprised the main approach to preventing disease spread. Important considerations for both diagnosing active infections and serologic testing include sample collection, accuracy and throughput of the detection method, experimental conditions, and accessibility of equipment required of various testing strategies. ${ }^{21}$ The complexity of controlling the emergence of a novel pathogen and worldwide pandemic posed extraordinarily difficult challenges for the rapid development of diagnostic tests that also allow for wide and efficient accessibility of testing. Testing for transmissible infections has predominantly involved the detection of viral RNA and viral antigens, which have been presented in depth in recent reviews. ${ }^{10,22}$ Collection methods have spanned a wide variety of sample types, including NP swabs, saliva tests, and many other mechanisms.

Of commercially available diagnostic tests, reverse transcription-polymerase chain reaction (RT-PCR) assays were developed early in the pandemic, and although they remain the most reliable available method of testing, raise challenges of efficiency and accessibility. ${ }^{10}$ RT-PCR is a highly sensitive bioassay that enables detection and quantification of RNA, a nucleic acid important for regulating gene expression that isfound in all cells and many viruses. Lead times of several days are common for commercial use of RT-PCR COVID19 tests, which require specialized training using expensive laboratory equipment that limit their application for point-ofcare (POC) use. The demand to reduce the resources and time needed for diagnostic results have led to significant developments in rapid testing enabled by viral antigen detection. ${ }^{23}$ While fewer commercial tests are available, rapid antigen testing can provide simple readouts in minutes from a nasal swab. Antigen testing works by detecting specific molecules or molecular structures on a pathogen (like SARS-CoV-2). In general, these assays lack the same specificity and efficacy of RT-PCR detection, primarily because viral loads need to be quite high in order to reach detectable levels on these kinds of tests; however, their ease of use and short turn-around time for a result have made a case for COVID-19 testing strategies utilizing rapid testing for initial screening to reduce demand for RT-PCR tests. ${ }^{10,23}$

Multidisciplinary approaches at the interface of materials research and biology have afforded novel biosensors with the potential to improve the accessibility and speed of COVID19 diagnostics compared to RT-PCR tests. The incorporation of carbon nanotubes, ${ }^{24,25}$ graphene, ${ }^{26,27}$ and many other types of nanomaterials in biosensors has drawn significant interest for their ability to provide highly sensitive diagnostic tools. ${ }^{28}$ Many of these technologies began in academic research labs, but several have been translated to POC technologies for several conditions and diseases ranging from cancer, diabetes, and infectious diseases. ${ }^{29}$ A major motivation behind emerging materials research for COVID-19 testing is the design of biosensors that are well suited for POC diagnostics, where sensitivity, mobility, and accessibility of materials are important considerations to enable rapid and widespread application 
including in resource-limited settings. ${ }^{30,31}$ In this issue, Furst et al. present recent developments in the area of electrochemical biosensors for the detection of active infections caused by emerging pathogens. Of particular interest are bioelectrochemical platforms that enable functional biosensors with enhanced specificity and sensitivity in addition to revealing fundamental insights into host-pathogen interactions.

Accessible diagnostic tools for active infections are necessary for mitigating the COVID-19 pandemic and future emerging pathogens, but robust serologic assays are also a critical factor in monitoring and controlling disease spread. In this issue, Sadtler et al. present a global perspective on materials concerns for accurate and efficient serologic testing to evaluate exposure and immunity in populations across the world, which has been a method employed by the NIH to study and track SARS-CoV-2. Serologic testing enables quantification of antibodies within serum derived from small volumes of blood; antibodies are specific proteins that are produced by B-cells in response to a specific antigen and provide a very clear and accurate method for understanding prior infection or immunity. ${ }^{32}$ With the development of serologic assays for COVID-19, including lateral flow immunoassays and enzymelinked immunosorbent assays (ELISA), ${ }^{21,22}$ this article discusses the importance of considering all key factors that affect assay reliability in order to provide accurate representations of seroprevalence in different populations worldwide.

\section{Materials science for the treatment and prevention of COVID-19}

One of the most noteworthy combatants of COVID-19 over the last 18 months has been the massive effort, pivoting, and collaboration demonstrated by researchers in virology, epidemiology, immunology, public health, and clinical settings across the globe to establish our baseline understanding of the disease. ${ }^{33}$ Without this information, the downstream efforts in materials science would arguably not be as effective or important, particularly for developing treatments or prevention measures. The role and impact of materials science and innovation on tackling COVID-19-related pandemic challenges has been thoroughly reviewed. ${ }^{34-37}$ In this issue, we provide an additional fresh overview and outlook of changes in treatment and prevention development.

As the SARS-CoV-2 virus enters the body, glycosylated spike proteins surrounding the virus directly bind to the host cell receptor angiotensin-converting enzyme 2 (ACE2), which mediates viral cell entry. ${ }^{38}$ Subsequent to viral replication and subgenomic RNA synthesis, viral proteins are translated into the host cell and consequently form mature virions. Virions are transported to the cell surface in vesicles and released via exocytosis; ${ }^{39}$ vesicles and infected host cells express antigens which can be targeted with antibody treatments. Antibody treatments for SARS-CoV-2 gained media notoriety in October 2020 when former US President Donald J. Trump was given an experimental Regeneron monoclonal antibody cocktail treatment, which has now been approved for more widespread use. ${ }^{40}$ While antibody-based therapeutics are not new, recent advances in nanotechnology and biomaterials have enhanced antibody targeting to the virus, led to development of broad-spectrum antibody treatments against SARS-CoV2-related cytokine storms, and have enhanced biomimetic properties of these therapies, which have also been used to develop host cell decoys. ${ }^{41}$

Conjugating antibodies to various biomaterials - nanoparticles, polymers, hydrogels, scaffolds, and extracellular vesicles - has been an effective strategy for increasing biodistribution, antibody homing, and pharmokinetics in vivo. ${ }^{42}$ In addition to enhancing the efficacy of antibody therapeutics, nanoparticles have shown efficacy in creating a decoy to absorb the virus and mitigate virus-related immune disorder. Biomaterials have played a major role in enhancing existing therapeutics to treat SARS-CoV-2 infections, while also opening up new avenues for innovation. In this issue, Wayne et al. provides a robust overview of the historical context of this pandemic, the role of material-based strategies to treat SARSCoV-2 infections and their effectiveness, and introduces developments in infection prevention, including vaccines.

Lipid nanoparticle-based mRNA vaccines, such as those created and approved by the FDA under emergency use authorization (EUA) by Moderna and Pfizer-BioNTech, have been a groundbreaking and highly effective technology for preventing further spread of the SARS-CoV-2 virus. ${ }^{43-46}$ Outside of the lipid nanoparticle-based mRNA vaccines or adenovirus-based vaccine from Janssen (Johnson \& Johnson) under EUA in the USA, others have investigated biomaterialbased immunomodulation to act as artificial lymph nodes or even as a novel vaccine delivery strategy. ${ }^{47}$ Langellotto et al. developed mesoporous silica rods to provide sustained release of GM-CSF and adjuvant monophosphoryl lipid A loaded with SARS-CoV-2 spike protein S1 and S2, nucleocapsid protein, and receptor binding domain antigens. ${ }^{48}$ This singleshot biomaterial-based vaccine facilitated a robust humoral response eliciting SARS-CoV-2 antibody titers in a murine model. While this technology is still being investigated in the lab, this biomaterial-based vaccine not only demonstrates a novel approach for preventing SARS-CoV-2 infection, but also shows significant potential for developing related vaccines against other infections. In this issue, Fenton et al. highlights the years of development that preceded the lipid nanoparticle-based mRNA vaccines that we have today, along with an outlook on the use of lipid nanoparticle technology. Collectively, biomaterial-based platforms have been imperative in the development of tools to meet the needs of the COVID-19 pandemic, and have also generated foundational technologies with potential to impact future global health efforts.

\section{Conclusions and outlook}

This issue of MRS Bulletin highlights innovations and provides thorough reviews across the field of materials science that have made direct (or foundational) contributions to pandemic-fighting measures since the outbreak of SARS-CoV-2. 
Features include discussions of novel improvements to NP swap testing and design, filter design in filter facepiece respirators, diagnostic testing to assess current and prior infection, and immunity, along with treatment and vaccine development for the infection prevention. The work reviewed in this issue also lays the groundwork for materials-relevant development that can combat - or prevent - future global health crises. Importantly, much of this innovation has been celebrated and embraced by the scientific and clinical communities alike; however, adoption of new technologies, particularly vaccines, by the general public has not reached the same level of acceptance. This emphasizes the need for greater transparency in the research, development, and translation process and importance of clear scientific communication by expertsfrom how our masks are created and tested, to the generation of a sophisticated vaccine to help prevent severe infection.

\section{Acknowledgments}

Portions of this work were performed under the auspices of the US Department of Energy by Lawrence Livermore National Laboratory under Contract No. DE-AC52-07NA27344.

\section{Conflict of interest}

On behalf of all authors, the corresponding author states that there is no conflict of interest.

\section{References}

1. World Health Organization, WHO Coronavirus (COVID-19) Dashboard (2021). https:// COVID19.who.int/

2. A. Tooker, M.L. Moya, D.N. Wang, D. Freeman, M. Borucki, E. Wheeler, G. Larsen, M. Shusteff, E.B. Duoss, C.M. Spadaccini, MRS Bull. 46(9), 2021. https://doi.org/10. 1557/s43577-021-00170-9.

3. P.L. Wang, A. Roschli, M. Parans Paranthaman, M. Theodore, C.L. Cramer, C. Zangmeister, Y. Zhang, J.J. Urban, L. Love, MRS Bull. 46(9), 2021. https://doi.org/10.1557/ s43577-021-00173-6.

4. J.E. Manning, P.E. Duffy, D. Esposito, K. Sadtler, MRS Bull. 46(9), 2021. https://doi. org/10.1557/s43577-021-00167-4

5. M.C. Machado, M. Zamani, S. Daniel, A.L. Furst, MRS Bull. 46(9), 2021. https://doi. org/10.1557/s43577-021-00172-7

6. Y. Lee, M. Ng, K. Daniel, E. Wayne, MRS Bull. 46(9), 2021. https://doi.org/10.1557/ s43577-021-00185-2

7. J.L. Andresen, O.S. Fenton, MRS Bull. 46(9), 2021. https://doi.org/10.1557/ s43577-021-00169-2

8. T.R. Mercer, M. Salit, Nat. Rev. Genet. 22, 415 (2021). https://doi.org/10.1038/ s41576-021-00360-w

9. Centers for Disease Control and Prevention, COVID Data Tracker. https://covid.cdc. gov/covid-data-tracker/\#trends dailytrendscases (2021)

10. T. Kilic, R. Weissleder, H. Lee, Science 23, 101406 (2020). https://doi.org/10. 1016/j.isci.2020.101406

11. Y.Y. Zuo, W.E. Uspal, T. Wei, ACS Nano 14, 16502 (2020). https://doi.org/10.1021/ acsnano.0c08484

12. Centers for Disease Control and Prevention, 42 C.F.R. Part 84 Respiratory Protective Devices (2021). https://www.cdc.gov/niosh/nppt//topics/respirators/pt84abs2. html

13. 73M. Comparison of FFP2, KN95, and N95 Filtering Facepiece Respirator Classes. (2021)

14. R.J. Fischer, D.H. Morris, N. van Doremalen, S. Sarchette, M.J. Matson, T. Bushmaker, C.K. Yinda, S.N. Seifert, A. Gamble, B.N. Williamson, S.D. Judson, E. de Wit, J.0. Lloyd-Smith, V.J. Munster, MedRxiv. (2020). https://doi.org/10.1101/2020.04.11. 20062018

15. A. Kumar, S.B. Kasloff, A. Leung, T. Cutts, J.E. Strong, K. Hills, G. Vazquez-Grande, B. Rush, S. Lother, R. Zarychanski, J. Krishnan, MedRxiv. (2020). https://doi.org/10. 1101/2020.04.05.20049346

16. T.L. Massey, M.K. Borucki, S.Y. Paik, K.W. Fuhrer, M. Bora, S.R. Kane, R.-U.M. Haque, S.H. Baxamusa, Ann Work Expo Health (2021). https://doi.org/10.1093/ annweh/wxab020
17. US Food and Drug Administration, Decontamination System EUAs for Personal Protective Equipment (2021). https://www.fda.gov/medical-devices/coronavirus-disea se-2019-covid-19-emergency-use-authorizations-medical-devices/decontaminationsystem-euas-personal-protective-equipment

18. T. Vora, A. Bhattacharya, S. Ghosh, K. Gowda, N. Dhanaki, R. Gala, N. Dubey, V. Raut, V.K. Singh, G. Prakash, S.G. Laskar, M. Sengar, G. Chinnaswamy, C.S. Pramesh, MedRxiv. (2020). https://doi.org/10.1101/2020.09.28.20203067

19. US Food and Drug Administration, Transition from Use of Decontaminated Disposable Respirators - Letter to Health Care Personnel and Facilities (2021). https:// www.fda.gov/medical-devices/letters-health-care-providers/fda-recommends-trans ition-use-decontaminated-disposable-respirators-letter-health-care-personnel-and 20. (NVBL), N. V. B. L. (2021). https://science.osti.gov/nvbl

21. N. Ravi, D.L. Cortade, E. Ng, S.X. Wang, Biosens. Bioelectron. 165, 112454 (2020). https://doi.org/10.1016/.j.bios.2020.112454

22. L.J. Carter, L.V. Garner, J.W. Smoot, Y. Li, Q. Zhou, C.J. Saveson, J.M. Sasso, A.C. Gregg, D.J. Soares, T.R. Beskid, S.R. Jervey, C. Liu, ACS Cent. Sci. 6, 591 (2020). https://doi.org/10.1021/acscentsci.0c00501

23. R.W. Peeling, P. Olliaro, D. Boeras, N. Fongwen, Lancet Infect. Dis. (2021). https:// doi.org/10.1016/s1473-3099(21)00250-4

24. S. Jeong, E. González-Grandío, N. Navarro, R.L. Pinals, F. Ledesma, D. Yang, M.P. Landry, ACS Nano 15, 10309 (2021). https://doi.org/10.1021/acsnano.1c02494

25. R.L. Pinals, F. Ledesma, D. Yang, N. Navarro, S. Jeong, J.E. Pak, L. Kuo, Y.C. Chuang, Y.W. Cheng, H.Y. Sun, M.P. Landry, Nano Lett. 21, 2272 (2021). https://doi. org/10.1021/acs.nanolett.1c00118

26. J. Sengupta, A. Adhikari, C.M. Hussain, Carbon Trends 4, 100072 (2021). https:// doi.org/10.1016/j.cartre.2021.100072

27. G. Seo, G. Lee, M.J. Kim, S.-H. Baek, M. Choi, K.B. Ku, C.-S. Lee, S. Jun, D. Park, H.G. Kim, S.-J. Kim, J.-O. Lee, B.T. Kim, E.C. Park, S.I. Kim, ACS Nano 14, 5135 (2020). https://doi.org/10.1021/acsnano.0c02823

28. S. Sharma, S. Saini, M. Khangembam, V. Singh, IEEE Sens. J. 21, 5598 (2021). https://doi.org/10.1109/JSEN.2020.3036748

29. N.M. Noah, P.M. Ndangili, J. Anal. Methods Chem. (2019). https://doi.org/10.1155/ 2019/2179718

30. A. Parihar, P. Ranjan, S.K. Sanghi, A.K. Srivastava, R. Khan, ACS Appl. Bio Mater. 3, 7326 (2020). https://doi.org/10.1021/acsabm.0c01083

31. S. Suleman, S.K. Shukla, N. Malhotra, S.D. Bukkitgar, N.P. Shetti, R. Pilloton, J. Narang, Y. Nee Tan, T.M. Aminabhavi, Chem. Eng. J. 414, 128759 (2021). https://doi. org/10.1016/j.cej.2021.128759

32. A.K. Winter, S.T. Hegde, Lancet Infect. Dis 20, 758 (2020). https://doi.org/10.1016/ s1473-3099(20)30322-4

33. T. Carvalho, F. Krammer, A. Iwasaki, Nat. Rev. Immunol. 21, 245 (2021). https:// doi.org/10.1038/s41577-021-00522-1

34. D. Chakhalian, R.B. Shultz, C.E. Miles, J. Kohn, J. Biomed. Mater. Res. A 108, 1974 (2020). https://doi.org/10.1002/jbm.a.37059

35. T. Colombani, Z.J. Rogers, L.J. Eggermont, S.A. Bencherif, Emerg. Mater. (2021). https://doi.org/10.1007/s42247-021-00171-z

36. Y.N. Ertas, M. Mahmoodi, F. Shahabipour, V. Jahed, S.E. Diltemiz, R. Tutar, N. Ashammakhi, Emergent Mater. (2021). https://doi.org/10.1007/s42247-021-00165-X 37. Z. Tang, N. Kong, X. Zhang, Y. Liu, P. Hu, S. Mou, P. Liljestrom, J. Shi, W. Tan, J.S. Kim, Y. Cao, R. Langer, K.W. Leong, O.C. Farokhzad, W. Tao, Nat. Rev. Mater. (2020). https://doi.org/10.1038/s41578-020-00247-y

38. M. Letko, A. Marzi, V. Munster, Nat. Microbiol. 5, 562 (2020). https://doi.org/10. 1038/s41564-020-0688-y

39. A.R. Fehr, S. Perlman, Methods Mol. Biol. 1282, 1 (2015). https://doi.org/10.1007/ 978-1-4939-2438-7_1

40. D.M. Weinreich, S. Sivapalasingam, T. Norton, S. Ali, H. Gao, R. Bhore, B.J. Musser, Y. Soo, D. Rofail, J. Im, C. Perry, C. Pan, R. Hosain, A. Mahmood, J.D. Davis, K.C. Turner, A.T. Hooper, J.D. Hamilton, A. Baum, C.A. Kyratsous, Y. Kim, A. Cook, W. Kampman, A. Kohli, Y. Sachdeva, X. Graber, B. Kowal, T. DiCioccio, N. Stahl, L. Lipsich, N. Braunstein, G. Herman, G.D. Yancopoulos, N. Engl. J. Med. 384, 238 (2021). https://doi.org/10. 1056/NEJMoa2035002

41. Q.F. Meng, R. Tian, H. Long, X. Wu, J. Lai, O. Zharkova, J.W. Wang, X. Chen, L. Rao, Adv. Mater. 33, e2100012 (2021). https://doi.org/10.1002/adma.202100012

42. X. Huang, J.Z. Williams, R. Chang, Z. Li, C.E. Burnett, R. Hernandez-Lopez, I. Setiady, E. Gai, D.M. Patterson, W. Yu, K.T. Roybal, W.A. Lim, T.A. Desai, Nat. Nanotechnol. 16, 214 (2021). https://doi.org/10.1038/s41565-020-00813-z

43. R. Verbeke, I. Lentacker, S.C. De Smedt, H. Dewitte, J. Control. Release 333, 511 (2021). https://doi.org/10.1016/j.jconrel.2021.03.043

44. F. Wang, R.M. Kream, G.B. Stefano, Med. Sci. Monit. 26, e924700 (2020). https:// doi.org/10.12659/MSM.924700

45. K.L. Swingle, A.G. Hamilton, M.J. Mitchell, Trends Mol. Med. 27, 616 (2021). https://doi.org/10.1016/j.molmed.2021.03.003

46. A. Khurana, P. Allawadhi, I. Khurana, S. Allwadhi, R. Weiskirchen, A.K. Banothu, D. Chhabra, K. Joshi, K.K. Bharani, Nano Today 38, 101142 (2021). https://doi.org/10. 1016/j.nantod.2021.101142

47. J. Zarubova, X. Zhang, T. Hoffman, M.M. Hasani-Sadrabadi, S. Li, Matter 4, 1528 (2021). https://doi.org/10.1016/j.matt.2021.02.025 


\section{IMPACT OF COVID-19 ON MATERIALS SCIENCE RESEARCH INNOVATION AND RELATED PANDEMIC RESPONSE}

48. F. Langellotto, B.T. Seiler, J. Yu, M.J. Cartwright, D. White, C. Yeager, M. Super, E.J. Doherty, D.H. Barouch, D.J. Mooney, A rapidly adaptable biomaterial vaccine for SARS-CoV-2. bioRxiv. (2020). https://doi.org/10.1101/2020.07.07.192203

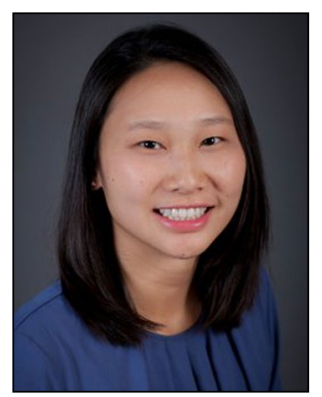

Crystal Chu is an assistant professor in the Department of Chemistry at Lehigh University. She received her BS degree from the University of California, Berkeley, in 2012, and PhD degree from the California Institute of Technology in 2017. Prior to joining Lehigh, she was a National Institutes of Health Postdoctoral Fellow at the Massachusetts Institute of Technology. Her research combines organic chemistry, materials science, and bioengineering. Chu can be reached by email at ckc221@lehigh.edu.

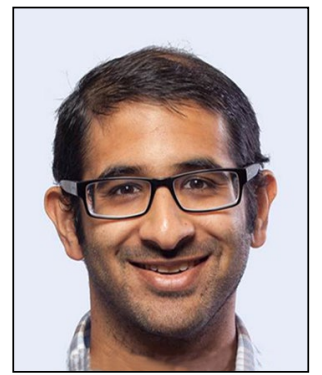

Salmaan Baxamusa is a staff scientist at Lawrence Livermore National Laboratory, where is a deputy group leader within the Materials Science Division. He received his BS degree from the University of California, Berkeley, in 2004, and $\mathrm{PhD}$ degree from the Massachusetts Institute of Technology in 2009. His research focuses on how material systems change as they interact with their environment, and Baxamusa develops materials processing techniques to prevent or control these changes. He received an R\&D100 Award in 2016. Baxamusa can be reached by email at baxamusa1@|lnl.gov.

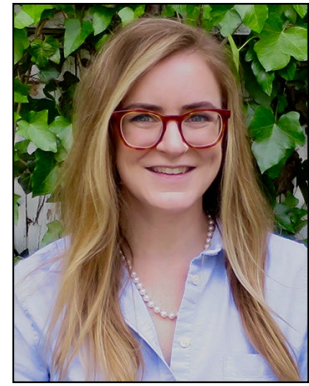

Claire Witherel is a University of Pennsylvania Provost Postdoctoral Research Fellow in the Department of Bioengineering. She received her PhD degree from Drexel University, studying innate immune-stromal cell crosstalk in the context of biomaterial-mediated fibrosis. Her research interests include the development of immunomodulatory hyaluronic acid-based hydrogels for the treatment of osteoarthritis in the knee. Witherel can be reached by email at withere!@seas.upenn.edu. 